\title{
De la phrase et d'un certain usage du participe présent propre aux romans de Claude Simon
}

\section{Philippe Forest}

\section{Q OpenEdition}

1 Journals

Édition électronique

URL : http://journals.openedition.org/ccs/832

DOI : $10.4000 /$ ccs.832

ISSN : 2558-782X

Éditeur :

Presses universitaires de Rennes, Association des lecteurs de Claude Simon

\section{Édition imprimée}

Date de publication : 31 décembre 2011

Pagination : 229-237

ISBN : 9782354121464

ISSN : 1774-9425

Référence électronique

Philippe Forest, « De la phrase et d'un certain usage du participe présent propre aux romans de Claude Simon », Cahiers Claude Simon [En ligne], 7| 2011, mis en ligne le 21 septembre 2017, consulté le 15 septembre 2020. URL : http://journals.openedition.org/ccs/832 


\section{De la phrase et d'un certain usage du participe présent propre aux romans de Claude Simon}

1. Est-ce qu'on sait jamais d'où viennent les livres, ses livres? On le découvre un peu une fois qu'on les a écrits, que l'on se retourne sur ce que l'on a fait et dont, jusque-là, on ignorait encore tout. $\mathrm{Ou}$ du moins l'essentiel.

Avant, une vague intention guide sans doute l'auteur mais la direction qu'elle lui indique est tellement incertaine que chaque page s'ajoutant à celles qui la précèdent, vient corriger l'ensemble et en modifier complètement la physionomie provisoirement informe. Il faut du temps pour que tout cela acquière une apparence plus ou moins acceptable. Et puis un moment arrive où, devant soi, l'espace du livre se matérialise mentalement dans son entièreté de telle sorte que les mots se disposent d'eux-mêmes en phrases qui, à leur tour, sécrètent autour d'elles les blocs des paragraphes se distribuant presque spontanément selon l'architecture subitement rendue visible des chapitres, ceux-ci s'enchaînant les uns aux autres jusqu'à ce que toute la place se trouve remplie et que, le sens porté à son seuil de saturation, il n'y ait plus rien qu'on soit en mesure d'ajouter.

Le roman est encore à écrire. Mais c'est comme s'il existait déjà. Qu'il se trouvait d'ores et déjà fini. Beaucoup d'autres en ont 
certainement fait la remarque avant moi : tout se passe comme si, depuis toujours, il avait été rédigé par quelqu'un à l'encre dite "sympathique ", celle de ces jeux désormais désuets auxquels se livraient les enfants d'autrefois. Ecrire revient alors à exposer la page apparemment blanche au révélateur du feu ou de l'acide. Un petit tour de prestidigitation puérile, un exercice de magie minuscule mais qui produit son effet, faisant surgir depuis l'épaisseur même du papier, à partir de la profondeur de la page, un message mystérieux dont, même si l'on en fut soi-même l'auteur, on devient un instant comme le lecteur incrédule et émerveillé, découvrant les mots que l'on a écrits comme s'ils avaient été tracés par la main d'un autre.

Même si l'image que j'emploie peut paraître indiquer le contraire (suggérant qu'un texte est déjà écrit qu'il ne reste plus qu'à faire apparaître), c'est à peu près ce que Claude Simon veut dire, je crois, lorsqu'il déclare : "Avant que je me mettre à tracer des signes sur le papier il n'y a rien, sauf un magma informe de sensations plus ou moins confuses, de souvenirs plus ou moins précis accumulés, et un vague - très vague - projet. »

Et puis : «C'est seulement en écrivant que quelque chose se produit, dans tous les sens du terme. Ce qu'il y a pour moi de fascinant, c'est que ce quelque chose est toujours infiniment plus riche que ce que je me proposais de faire. »

2. Ecrivant Le Siècle des Nuages, j'avais un très vague projet, celui de faire rentrer dans ce roman toute l'épaisseur du passé, tressant l'histoire de tous avec la mienne, avec pour fil directeur le destin d'un homme (mon père) lui-même lié à l'une des principales utopies de l'ère moderne (l'aéronautique). C'est tout.

Ligne par ligne, je pourrais expliquer comment, dès le prologue de l'ouvrage, j'ai progressivement compris que le roman trouverait sa forme à la stricte condition de ne pas renoncer à ce projet tout en manifestant la perpétuelle impossibilité de celui-ci, construisant et déconstruisant un récit qui signifierait ainsi que toute histoire 
n'existe jamais qu'à la façon d'une fiction inquiète où, au présent, celui qui écrit doit prendre acte de son incapacité à dire ce que fut le passé et s'emploie malgré tout à rendre celui-ci en laissant sa pensée errer parmi tout un dédale d'hypothèses, de certitudes, de faits et d'énigmes mais en se refusant toujours, autant que faire se peut, à occuper cette position de surplomb (ce que je nomme : «le confort de l'impensable futur ") d'où tout apparaît selon la perspective falsificatrice d'une vision d'après-coup.

Une telle conception du temps (au demeurant assez banale et aisément transposable dans le langage ressassé de certaines philosophies de l'histoire), je ne la possédais pas a priori comme s'il s'était agi d'en déduire ensuite une illustration par le récit. Ce sont mes propres phrases qui me l'ont découverte, enseignée à mesure que je les concevais. Prenant une ampleur que je ne prévoyais pas afin d'envelopper ces grands blocs de temps tombés de nulle part et entre lesquels n'existe aucune forme de continuité visible, se compliquant d'incises et de parenthèses pour que chaque affirmation puisse se trouver précisée, nuancée voire contredite au sein d'un même paragraphe, permettant ainsi au récit d'explorer successivement - et même simultanément toutes les pistes divergentes selon lesquelles se déploie ce que Joyce, dans Ulysse, appelle "l'infinie possibilité des possibles ». Recourant aux formes verbales qu'il faut afin que le texte parvienne à glisser sans cesse du passé au présent ou du présent au passé et laisse le lecteur un peu dans l'incertitude, ne sachant plus très bien ni quand l'action se situe ni qui en est le héros et faisant ainsi se dérouler sous ses yeux le drame d'une intrigue assez intemporelle et assez impersonnelle pour qu'elle puisse susciter sur la page le simulacre d'une scène suffisamment vaste pour recevoir en elle toutes les histoires du monde.

D'où les phrases interminables du Siècle des Nuages disposées en paragraphes à l'apparence de pavés disjoints où le récit se relance continuellement par l'usage insistant du participe présent. Comme une manière de retrouver, avec les moyens du bord et dans la langue du roman d'aujourd'hui, quelque chose, sans doute, d'une écriture épique se développant en longues laisses rythmées d'une lancinante rime en «-ant». 
3. J'avais un vague projet et, pour avouer toute la vérité, une sorte de modèle aussi dont j'ai pris conscience dès les premières pages et qui a certainement orienté toute la suite du livre.

Faulkner : pour la manière non pas inimitable mais inégalée dont il fait progressivement apparaître toute la matière du temps dans son irréductible opacité sous les yeux du lecteur, ne distillant à ce dernier que très parcimonieusement les informations qui lui seraient pourtant indispensables à la compréhension de l'intrigue et, en général, bien longtemps après le moment où il aurait été logique de les lui fournir, faisant ainsi résonner en tumulte à ses oreilles le bruit et la fureur de ce conte d'idiot qu'est la vie. Démontrant alors que l'histoire des hommes n'existe jamais que sous l'apparence des récits confus, contradictoires, incomplets que lui donnent ceux-ci et dont le roman peut seulement, dans sa formidable emphase, rendre la rumeur retentissant pour rien dans le temps.

Faulkner, et plus précisément ce livre de lui qui s'intitule $R e$ quiem pour une nonne et dont la spécificité tient à ce qu'il noue une intime affaire de deuil (celle de Temple Drake à l'enfant assassinée) avec la chronique récapitulée du Yoknapawtapha (celleci s'élargissant au point de contenir toute l'histoire de l'humanité, depuis son surgissement sur une terre vierge jusqu'au vertige de la modernité projetant la civilisation dans le précipice d'un perpétuel lendemain). Et plus particulièrement encore le dernier acte du roman pour le prodige poétique auquel l'auteur y parvient, Faulkner tendant sa phrase de sorte qu'une ligne vienne relier les époques les plus éloignées, corde vibrant à une fréquence inouïe, exprimant cette extraordinaire accélération des choses au sein de laquelle toutes elles défilent, semblent s'engendrer les unes les autres, et que la prose ne peut rendre qu'à la condition de renoncer aux structures ordinaires de sa syntaxe et, recourant à des formes bien plus recevables en anglais que ne l'est le participe présent du français, le texte se trouvant scandé en des paragraphes qui sonnent comme des strophes, traduire la précipitation même de la vie vers le vide qui l'appelle et au sein duquel elle laisse cependant la dérisoire et splendide inscription de son témoignage: "Going fast now... That 
fast, now... That fast, that rapid... Then gone... Moving faster and faster... "La page du Siècle des Nuages (la manière dont elle est composée), sa phrase (ses méandres, son rythme) avec ses participes présents viennent de là.

4. A Claude Simon, je n'ai pensé qu'ensuite. Une fois le livre terminé, un soupçon m’est venu. L'idée qu'existait en français et chez un auteur que j'avais lu autrefois quelque chose d'assez comparable à ce dont j'avais cru trouver la formule chez Faulkner. J'y ai songé quand j'ai été tenté de recomposer une dernière fois le roman, d'abandonner la linéarité apparente selon laquelle s'organisaient tous ses chapitres, de mélanger la chronologie de manière à donner au lecteur ce vrai sentiment de tournis que procure le mouvement de toupie d'un avion qui part en vrille et dont la chute devient l'axe autour duquel s'accomplit la révolution vertigineuse de l'espace et du temps.

J'ai renoncé à cette idée. Peut-être par paresse ou par lâcheté. Ou bien : par réalisme. Il ne faut pas trop en demander au lecteur d'aujourd'hui. C'est ce que je me dis quand je constate quelles violentes réactions de rejet suscite désormais tout texte un tout petit peu écrit : si une phrase dépasse les deux ou trois lignes avec une structure un peu plus étoffée que le simple «sujet-verbe-complément ", si un roman ne s'exprime pas selon les normes stéréotypées de la langue journalistique la plus pauvre, il a toutes les chances de passer pour « illisible ». Et donc pour " mal écrit ».

Par paresse ou par lâcheté, par calcul ou bien par manque de talent, ce que j'ai fait avec Le Siècle des Nuages est pourtant d'une simplicité assez enfantine par rapport à ce que Claude Simon a tiré de ses plus grands romans. C'est lui, bien sûr, qui a eu raison. A l'heure où Michel Houellebecq et Amélie Nothomb passent pour les écrivains majeurs de la littérature française, je me demande parfois combien de personnes peuvent encore lire Histoire ou La Bataille de Pharsale. Je me souviens très précisément d'avoir entendu, à l'occasion d'une des émissions les plus populaires de la radio nationale, c'était il y a dix ans, un chroniqueur littéraire déjà très en vue et 
dont les avis font désormais autorité sur la toile, déclarer à propos du dernier roman de Claude Simon, Le Tramway, qu'il ne s'agissait certainement pas d'un livre que l'on songerait à offrir à l'un de ses amis. Chacun a les amis qu’il mérite.

5. J'ai lu à peu près tous les romans de Claude Simon quand j'avais vingt ans. C'était donc il y a longtemps maintenant. Je ne les avais pratiquement jamais réouverts depuis et jusqu'à ce que me vienne le soupçon tardif que je leur devais sans doute davantage que je ne l'imaginais.

\section{De fait.}

Je dois bien admettre - je n'ai aucune difficulté à le faire - que cette langue dans laquelle s'exprime Le Siècle des Nuages et dont j'avais eu le sentiment qu'elle s'inventait d'elle-même, appelée par le vague projet que je m'étais fixé, peut légitimement apparaître comme la forme atténuée, de manière à souscrire un peu aux pauvres exigences de lisibilité qui s'imposent au romancier d'aujourd'hui, de celle, plus audacieuse et magnifique, que parle, dans sa prodigieuse poésie, l'œuvre de Claude Simon.

Dès le début du Vent, l'idée exprimée que raconter, " c'est un peu comme si on essayait de recoller les débris dispersés, incomplets, d'un miroir, s'efforçant maladroitement de les réajuster, n'obtenant qu'un résultat incohérent, dérisoire, idiot. " L'entêtement, malgré tout, à progresser au sein d'un tel chaos de manière à produire de celui-ci une représentation impossible par laquelle, comme on le lit dans La Route des Flandres, on touche peut-être à une "réalité plus réelle que le réel ». Dans ce même roman, les longues phrases qui donnent au lecteur le sentiment que l'on avance à tâtons dans le temps, se frayant un chemin vers nulle part dans l'immense nuit de la vie, tournant en rond, rebroussant sa route, se heurtant toujours aux mêmes spectacles de sidération désolée. Avec La Bataille de Pharsale et un peu partout ailleurs, ces participes présents qui décrochent la phrase de toute temporalité, étirant la durée d'un récit qui se suspend, "Achille immobile à grands pas », et dont personne 
ne peut plus dire vraiment à qui, à quoi elle se rapporte. Histoire, surtout, de tous les livres de Claude Simon celui auquel va ma préférence, sa formidable chute, le dernier mot du " moi " avec son point d'interrogation comme si celui qui écrit n'existait enfin qu'à la façon de l'effet très tardif et toujours incertain de cela qu'il a écrit. Ou encore cette manière si caractéristique de toujours montrer que ce qui est ne se situe nulle part sinon dans le récit qu'on en fait et qui culmine splendidement dans ce miroitement de la réalité et de la fiction se convertissant perpétuellement l'une en l'autre qu'organisent Triptyque et Leçon de choses ou bien dans le jeu plus ouvertement autobiographique des grands livres de la fin.

Et ainsi de suite.

6. Je croyais imiter Faulkner. Sans le savoir, je faisais peut-être du Claude Simon. Je le veux bien. Ce sont des choses qui arrivent. Et elles se passent toujours de façon plus complexe que ne le veut le dépistage un peu policier de ce que la vieille critique appelait " les influences " et que l'on baptise aujourd'hui du nom plus savant d' "intertextualité ». Le temps de la littérature n'est pas davantage linéaire que celui de l'histoire. Il se parcourt dans tous les sens à la fois, se ramifie en chemins parallèles qui se croisent enfin, il se disperse, s'éparpille. On peut en remonter aussi bien qu'en descendre le cours.

Le roman de Simon, c'est l'évidence, suppose celui de Faulkner dont il constitue souvent le pur démarcage (Le Vent ou, exemplairement, L'Herbe) à tel point que de nombreuses pages, surtout dans les premiers livres, y sonnent comme si elles étaient le résultat d'une sorte d'adaptation d'une langue à l'autre. Simon importe en français la phrase de Faulkner : la façon dont elle se développe, se déploie au point de s'égarer, se surcharge, digresse ou se répète (avec ses parenthèses) puis se reprend pour repartir dans une direction un peu nouvelle de sorte que le texte avance par spirales successives, par approximations progressives. D'où, je crois, aussi, mais je laisse les spécialistes en décider, le recours au participe présent qui transpose la forme en -ing et constitue, au fond, comme une sorte d'anglicisme 
à l'aide duquel le français se change en une langue vaguement étrangère, susceptible de produire poétiquement des effets de sens qui autrement lui seraient restés interdits. Les mêmes causes produisant les mêmes effets, il n'est pas inconcevable qu'un romancier d'aujourd'hui, important à son tour Faulkner dans sa phrase, parvienne à un résultat plus ou moins comparable.

Mais, en même temps, c'est l'évidence aussi, le roman de Simon expérimente une forme qui lui est propre et qui, tout en frappant par son extrême singularité, ne cesse de faire apparaître comment le grand roman moderne (tout grand roman authentiquement moderne) vise nécessairement à la restitution impossible d'une réalité qui ne se donne et ne se dérobe que sous la forme de fictions proliférantes, lacunaires, celles-ci se développant, ainsi que le dit Le Vent, autour de "quelque chose d'infime, de minuscule, insignifiant : rien du tout ", de manière à rendre visible ce grand mouvement qui agite le monde et lui confere comme le tremblement terrible et magnifique de la vie: «l'infatigable, permanente tempête se ruant sans trêve sous le ciel diaphane, s'exaspérant, s'enivrant de sa propre colère, de son inutile puissance, dépourvue de sens " - tout cela, on le note, s'exprimant impérativement au participe présent. Une sorte d'effet en retour s'exerce alors par lequel tous les livres, et notamment ceux de Faulkner, se reconfigurent, se recomposent pour acquérir l'apparence que leur confere la vision poétique souverainement initiée par un nouvel écrivain, en l'occurrence Simon, et par laquelle c'est toute l'histoire de la littérature passée qui se trouve dès lors reconsidérée et transformée selon la clarté que jette sur elle une ouvre d'après elle. Si bien qu'il n'y a rien d'étonnant, en somme, à ce qu'un romancier d'aujourd'hui n'accède aux écrivains d'avant-hier qu'à travers la réécriture à laquelle les ont soumis ceux d'hier.

Dans la mesure éminemment variable de ses moyens - je veux dire : de sa puissance poétique propre -, chaque auteur se trouve mis en demeure d'inventer toute la littérature à son tour. Au double sens qu'a ce verbe en français qui peut signifier aussi bien " découvrir »comme le fait l'archéologue ou l'explorateur - que " créer " - ainsi 
que s'y emploie l'ingénieur ou l'artiste. Et l'on sait que dans ce genre d'expériences, le résultat obtenu est souvent très différent de celui en quête duquel on s'était mis. Il arrive qu'un voyageur parte pour les Indes et qu'il se retrouve en Amérique. Plus modestement, il peut se produire qu'un romancier réécrive un autre œuvre que celle qu'il croyait s'être donnée pour modèle.

\section{Philippe FOREST}

Philippe Forest est né le 18 juin 1962 à Paris. Il est diplômé de l'Institut Politiques de Paris (1983) et docteur es lettres (1991). Il a vécu sept ans en Grande-Bretagne où il a enseigné la littérature française dans diverses universités (Edimbourg, Saint-Andrews, Cambridge, Londres). Actuellement professeur de littérature française à l'Université de Nantes, il est l'auteur de nombreux essais consacrés à la littérature et à l'histoire des courants d'avant-garde (notamment à l'histoire de Tel Quel) et il vient de faire paraître l'ouvrage, Beaucoup de jours. D'après Ulysse de James Joyce, aux éditions Cécile Defaut, (décembre 2011). Il a également séjourné au Japon et est lauréat de la Villa Kujoyama. Ses romans sont publiés chez Gallimard, L'Enfant éternel, 1997 (prix Femina du premier roman), Toute la nuit, 1999, Sarinagara, 2004 (prix Décembre), Le Nouvel Amour, 2007, Le Siècle des nuages, 2010. 